\title{
Structure Assembly by a Heterogeneous Team of Robots Using State Estimation, Generalized Joints, and Mobile Parallel Manipulators
}

\author{
Erik E. Komendera ${ }^{1}$, Shaurav Adhikari ${ }^{2}$, Samantha Glassner $^{3}$, Ashwin Kishen ${ }^{4}$, and Amy Quartaro ${ }^{5}$
}

\begin{abstract}
Autonomous robotic assembly by mobile field robots has seen significant advances in recent decades, yet practicality remains elusive. Identified challenges include better use of state estimation to and reasoning with uncertainty, spreading out tasks to specialized robots, and implementing representative joining methods. This paper proposes replacing 1) self-correcting mechanical linkages with generalized joints for improved applicability, 2) assembly serial manipulators with parallel manipulators for higher precision and stability, and 3) all-in-one robots with a heterogeneous team of specialized robots for agent simplicity. This paper then describes a general assembly algorithm utilizing state estimation. Finally, these concepts are tested in the context of solar array assembly, requiring a team of robots to assemble, bond, and deploy a set of solar panel mockups to a backbone truss to an accuracy not built into the parts. This paper presents the results of these tests.
\end{abstract}

\section{INTRODUCTION}

Autonomous assembly has the potential to reduce assembly time, expenses, and risks to human safety. Researchers aim to replicate the benefits of assembly line robots, which - in highly controlled and predictable environments precisely perform repetitive tasks in streamlined processes that have made advanced technology inexpensive and widely available. In the field, robots are expected to operate with added mobility and versatility, and must handle uncertainties in both sensor inputs and events in a dynamic environment. A compelling space application is on-orbit assembly, to mitigate costs and risks associated with astronaut extravehicular activities, reliance on large launch vehicles, and dependency on complicated deployment mechanisms [1]. Recent studies highlighted several applications for in-space assembly, including megawatt-class solar electric propulsion tugs (Figure 1A), large space telescopes [2]; artificial gravity vehicles, space docks, and in situ resource utilization [1].

Assembly tasks extend beyond snapping parts together, and include cutting and shaping parts, overcoming manufacturing errors, reusing or repurposing parts, making ad-hoc corrections to propagated errors, and inducing stresses to align parts. Often, the attachment of a subassembly requires several agents coordinating to complete the task. In a recent survey, Ardiny et al. [3] described several ongoing challenges

\footnotetext{
${ }^{1}$ E. E. Komendera is with the Structural Mechanics and Concepts Branch, NASA Langley Research Center, Hampton, VA 23681 erik.e.komendera@nasa.gov

${ }^{2}$ S. Adhikari is with the University of Michigan, Ann Arbor, MI 48109

${ }^{3} \mathrm{~S}$. Glassner is with Northeastern University, Boston, MA 02115

${ }^{4}$ A. Kishen is with the University of Pennsylvania, Philadelphia, PA 19104

${ }^{5}$ A. Quartaro is with the University of Texas, Austin, TX 78712
}

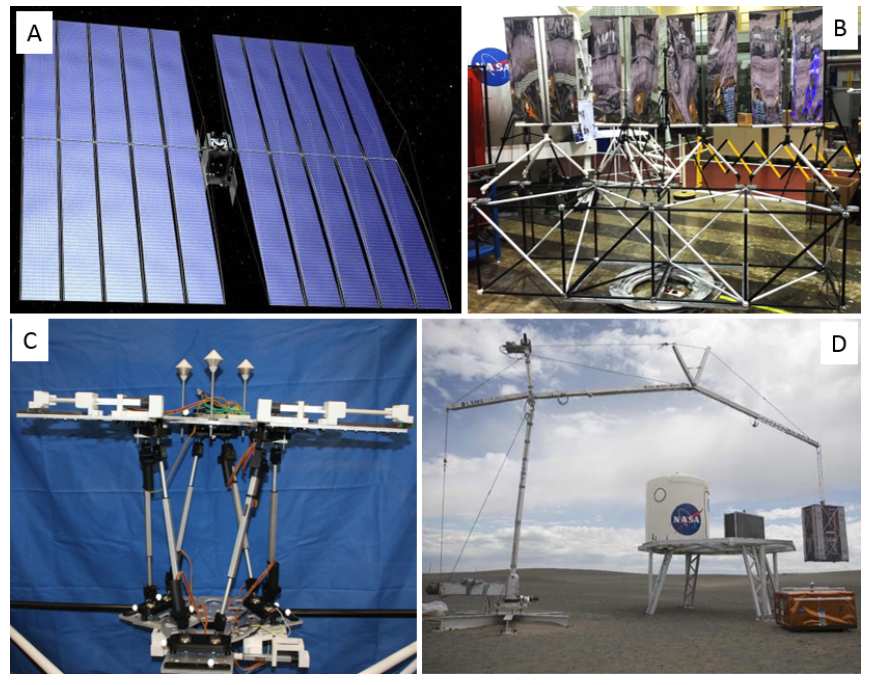

Fig. 1. A) Example application for practical autonomous robotic assembly: solar electric propulsion vehicles. B) Result of ground-based solar array mockup assembly trial performed by a team of heterogeneous robots, including C) a dexterous manipulator which positioned parts relative to one another, and D) a long reach manipulator responsible for transporting the dexterous manipulator and joining the solar panels to the backbone truss.

in autonomous mobile robotic assembly research; among them are:

- Employing external tracking and short-range relative localization for mobile robots while maintaining a map of the assembly site.

- Reasoning with uncertainties in the environment, the assembly materials, the agents, and unexpected events, instead of dodging these issues by imposing unrealistic constraints on the environment or sacrificing assembly precision.

- Improving robotic versatility and/or using cooperative heterogeneous robots. Complex and costly robots that can handle all tasks are at a higher risk of failure than a heterogenous team of simpler, specialized robots.

- Making adjustments as needed to parts and joining methods to correct tolerance, manufacturing, and propagated errors.

For space applications, the design and assembly of each part requires extensive planning, manufacturing, and checkout procedures, adding to time and cost. This hinders servicing, and prevents repurposing functional parts on derelict spacecraft. The advent of practical robotic in-space assembly will mitigate the need for deployment mechanisms and enable assembly using materials delivered by multiple launch 
vehicles. This reduction in complexity will lead to simplified common architectures, enabling interchangeable parts, and driving down costs.

In recent years, Langley Research Center has developed assembly methods to address some of these challenges [4] by distributing long reach manipulation tasks and precise positioning tasks between specialized agents, employing Simultaneous Localization and Mapping (SLAM) in the assembly workspace, using sequencing algorithms, and detecting and correcting errors. In [5], a heterogeneous team consisting of a long reach manipulator (LRM) and a dexterous manipulator (DM) collaborated to assemble and weld a flat truss made of titanium stock, which required the LRM and DM to collaborate in retrieving struts and nodes from a canister, the DM to position the nodes precisely, and the LRM to weld the struts and nodes together. In [6], a team of DMs collaborated in assembling a curved truss structure utilizing a SLAM method with only local measurements to position parts and to guide corrections to geometrical placement errors, reducing nodal positional error growth from $O\left(n^{3}\right)$ in the number of parts, to approximately constant nodal errors dominated by per-step process noise.

\section{A. Contribution}

This paper describes a process by which a heterogeneous team of robots collaborates to retrieve and reposition parts, assemble parts accurately without relying on mated joints, and modify the parts after joining. Specifically, this paper considers assembly using only generalized joints with featureless interfaces that are bonded together with techniques such as welding or using mortar, likely by a specialized robot. This enables the joining of parts that were not specifically mated, or were not machined with sufficient precision - for example, building hybrid structures using coarselymade in situ elements and pre-made manufactured parts. These joints permit variability in all six translational and rotational degrees of freedom, provided a gap between parts can be filled with material.

To achieve assembly with generalized joints, a DM must position and hold parts throughout the joining process. Such a manipulator must be stable and precise with high load capacity. Parallel manipulators are far better than serial manipulators for these requirements. Parallel manipulators have a significant disadvantage in range of motion, so an additional serial LRM assists in mobilizing the DM. This manipulator can also bond the joints. Unlike an all-in-one system, the LRM does not need precision and stability, and can even be made with compliant links such as the LRM presented here. Additionally, in lieu of self-correcting, mechanical interfaces, accurate assembly requires robust estimation of the states of all the parts and robots. This paper presents a general assembly algorithm for controlling the accurate assembly of structures where each part has realvalued positions and orientations, as opposed to discretized representations that ignore tolerances, backlash, strain, and propagating errors.

This method is tested in the context of assembling solar panels to a backbone truss (Figure 1B). Each robot must perform a wide variety of tasks: the DM (Figure 1C) must retrieve a stowed solar panel from storage, move to the backbone truss with assistance from the LRM, attach to the truss, and position the panel; the LRM (Figure 1D) must join the panel to the truss using an analogue of welding and deploy the panels into a functional configuration. This paper then describes the results of several hardware assembly trials, and discusses directions for future research.

\section{B. Related Work}

Assembly by quadrotors is attractive due to their functional workspace [7], and have been the subject of reinforcement learning study [8]. Other approaches focus on truss assembly, reconifiguration, and transversal [9], [10]. Visual feedback is used to identify and replace missing parts [11]. Foam deposition assembly is explored in [12], which is a notable exception to the use of mechanical joints. ETH Zurich has explored many approaches to robotic assembly, including the assembly of bricks whose as-built dimensions are measured and incorporated into the sequence planning [13], and the aerial assembly of tension bridges [14]. Industry looks to use mobile factory floor robots, leading to competitions such as the Airbus Shopfloor Challenge [15], which builds on peg-in-hole assembly [16], [17]. NASA has explored assembly for space applications; a team of three robots collaborated to join a part to a structure [18], a pair of robots precisely assembled beams using rigid motions [19], and an arm assembled and disassembled a telescope truss with reflector segments [20].

\section{Assembly With Generalized Joints}

The use of mated parts hinders the ability for corrections to be made if tolerances or manufacturing quality are poor. For example, if part $A$ has a manufacturing error leading to an interface position error, then a joined part $B$ will inherit that error. This error propagates through $B$, which adds its own error, and so on. With human agents, these kinds of errors can be easily seen through observation, and corrected through means such as ad-hoc part modification and inducing stresses on structures to line up matching features.

An additional concern in mechanical interfaces is backlash. In some cases backlash is irrelevant, but in spacecraft design and structures with heavy load requirements, backlash creates the potential for collision fatigue within joints, and impacts positioning and stability. Precise machining and spring-loaded joints mitigate backlash, but this complicates joint design and manufacturing.

One method for mitigating error propagation and eliminating backlash is to use interfaces that permit large allowances in the translation and rotation axes, and rigidizing the joint once conditions are satisfied. A general version of this kind of interface is a weldable gap between parts with featureless surfaces. For example, flat surfaces in contact can be aligned with up to three degrees of freedom - translation in two axes and rotation in one - with three extra degrees of freedom if space is permitted between the parts. 
TABLE I

QUALITATIVE COMPARISON OF SERIAL AND PARALLEL MANIPULATORS FOR PART ALIGNMENT DURING ASSEMBLY.

\begin{tabular}{|l|l|l|}
\hline Manipulator & Pros & Cons \\
\hline Serial & $\begin{array}{l}\bullet \text { Large workspace volume } \\
\bullet \text { Hand-over-hand mobility } \\
\text { with end-effectors }\end{array}$ & $\begin{array}{l}\bullet \text { Small payload due to } \\
\text { cantilever torque } \\
\bullet \text { More flexible }\end{array}$ \\
\hline Parallel & $\begin{array}{l}\bullet \text { Large payload capacity } \\
\text { due to stiff topology, high } \\
\text { gear ratio linear actuation } \\
\bullet \text { Less flexible }\end{array}$ & $\begin{array}{l}\bullet \text { Small workspace volume } \\
\bullet \text { Needs additional actua- } \\
\text { tion or assistance to be } \\
\text { mobile }\end{array}$ \\
\hline
\end{tabular}
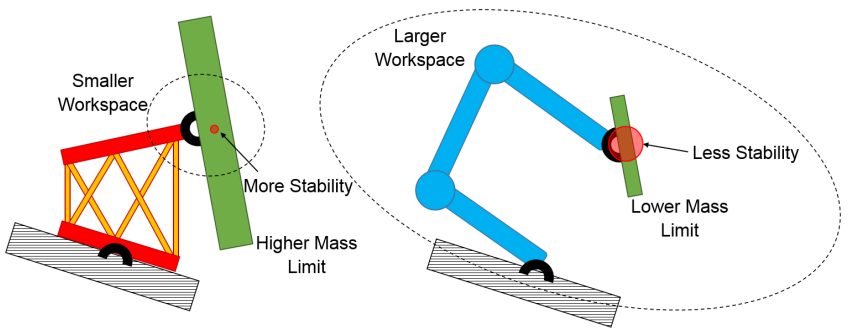

Fig. 2. Conceptual comparison between parallel (left) and serial (right) manipulators for assembly. The dotted ellipses represent the ranges of motion of the manipulators, and the pink circles represent instability under perturbations.

\section{A. Positioning Parts with Stewart Platforms}

Before welding, parts need to be aligned, or "jigged," by a manipulator. Due to their ubiquity and large workspace, serial manipulators are frequently used in industry, while parallel manipulators such as delta robots have been used as pick-and-place machines [21] in well-controlled environments. Another kind of parallel manipulator, the Stewart platform, has numerous advantages. Their large payload capacity has the potential to enable positioning of large volume and mass parts. Stewart platforms with sub-micron positioning precision can be built with off-the-shelf linear actuators [5]. The octrahedral topology of Stewart platforms increases stiffness and stability to facilitate joining methods without unwanted vibrations. Table I compares the two types of manipulators in the context of part alignment, and Figure 2 illustrates the concept. To the best of our knowledge, mobile parallel manipulators for assembly have never been considered.

\section{B. Heterogeneity with Long Reach Manipulators}

A system using dedicated Stewart platform DMs requires other agents to perform long reach tasks and locomotion. Mobile LRMs can be used to fill this void. They can either be mounted on a mobile base or have the ability to walk hand-over-hand. They can be fitted with swappable endeffectors to make them more versatile. Since they are not required to perform dexterous, high precision tasks, their design can be simplified. The Lightweight Surface Manipulation System (LSMS)[22], for example, is a 15 meter-long, tendon-actuated arm with passive compliance. An industrial assembly arm with similar reach would be more massive, would require large and powerful motors, and would be a
Sequential Assembly with State Estimation: $A, \bar{X}_{0}, \Sigma_{0}$

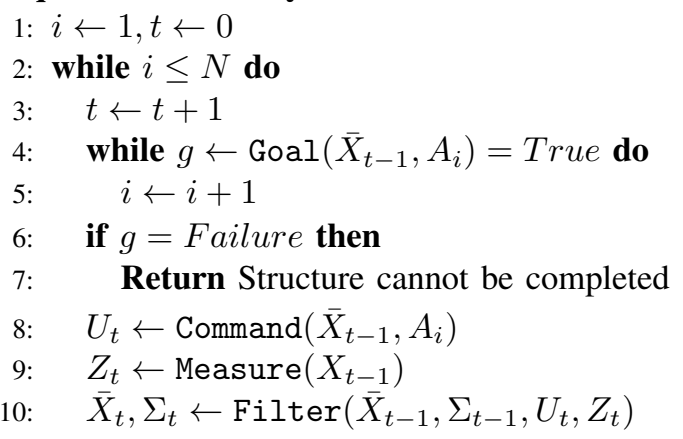

11: Return Structure complete

Fig. 3. Starting with the assembly steps $A$, and an initial estimate of the states of the parts and robots $\bar{X}_{0}$ with uncertainty $\Sigma_{0}$, this algorithm iterates over each step until each goal is met. Each iteration checks for goal status, commands the robots, then measures and updates the state estimates.

hazard to humans.

The DM's grasping tools have another use: by locking into the end effector of the LRM, the DM gains a form of assisted mobility. To reposition the DM, the LRM grossly positions the DM near an assembly site; the DM uses its fine positioning capability to attach to the assembly site and detach from the LRM. In addition, while attached to the LRM, the DM may act as a precise end-effector for the LRM. With passive compliance on the LRM, the risks of contact between the arm, the DM, and the structure can be reduced when the DM is simulatenously attached to the LRM and to the assembly site.

\section{Assembly with State Estimation}

The generality of the joints requires the structure definition contain real-valued positions and orientations for each part. Thus, the assembly robots must maintain a map of the entire structure and their own positions within. An assembly sequence algorithm operating with both measurement and actuation uncertainties must set goals that are achievable within the constraints of the metrology and positioning systems. The algorithm presented in Figure 3 is sequential, but can be modified to enable parallel assembly by permitting simultaneous steps in which some step $i$ can be started when precursor steps $\{i-a, i-b, \ldots\}$ have been completed. Task distribution becomes an instance of a job scheduling problem, beyond the scope of this paper, but will be considered in future work.

In general, at time $=t, S_{t}=\left[S_{1} \ldots S_{\sigma}\right]$ contains the state of the structure, where each part's state includes position, orientation, and assembly status ${ }^{1} . R_{t}=\left[R_{1} \ldots R_{\rho}\right]$ contains the state of the robots, with each state containing position, orientation, and other status details (e.g. DM is connected to LRM). The combined state is $X_{t}=\left[S_{t}, R_{t}\right]$. The state must be estimated as $\bar{X}_{t}$ with an uncertainty term $\Sigma_{t}{ }^{2}$. The control vector $U_{t}=\left[U_{1} \ldots U_{\rho}\right]$ contains all commands

\footnotetext{
${ }^{1}$ Specific details will vary with application.

${ }^{2} \Sigma_{t}$ may represent a covariance matrix for Kalman filters, or a particle set for particle filters.
} 
issued to the robots. $Z_{t}$ contains all measurements of the structure and the robots. $N$ is the number of steps needed to complete the structure ${ }^{3}, i$ the step index, $A_{i}$ the step goal, and $A=\left[A_{1} \ldots A_{N}\right]$. Goal $\left(\bar{X}_{t-1}, A_{i}\right)$ is a function that returns True if the step goal has been achieved, False if not, and Failure if the goal cannot be met, which permits termination of the algorithm. Command $\left(\bar{X}_{t-1}, A_{i}\right)$ commands the robots and returns $U_{t}$. Command handles any required adjustments. Measure $\left(X_{t-1}\right)$ collects measurements from the environment, and Filter $\left(\bar{X}_{t-1}, \Sigma_{t-1}, U_{t}, Z_{t}\right)$ estimates the state. The assembly sequence starts with a priori estimates for the state, covariance, and initial controls.

\section{SOlAR ARrAy ASSEMBly EXPERIMENT}

Solar electric propulsion (SEP) spacecraft are an ideal target for in-space assembly. Electric thrusters for space missions require $100 \mathrm{~kW}$ to $400 \mathrm{~kW}$ of power to operate, which requires hundreds of square meters of panel surface area [1]. These panels require supporting structures with high strength and stiffness. The ability to assemble, disassemble, and repair individual panels has the potential to expand the operational lifetime of such a vehicle. NASA and the National Institute of Aerospace recently hosted a university competition for SEP mission designs requiring on-orbit assembly [23].

A series of solar array assembly trials were performed using the methods presented in this paper. A heterogeneous team of robots collaborated autonomously to accurately assemble and deploy a mockup solar array consisting of stowed solar panels and a backbone truss. The DM in this experiment is an iteration of the Intelligent Precision Jigging Robot (IPJR). The LRM is the LSMS ${ }^{4}$. To give the LSMS an extra degree of freedom, the truss rests on an autonomous turntable. Figure 4 shows all robots and parts.

A Vicon metrology system [24] measures the positions of all objects. The primary goal was the successful joining and deployment of all panels. The secondary goals were achieved when the panels were measured to be within a $10 \mathrm{~mm}$ error box centered on the goal with a maximum of 3 degrees of error on each axis in an axis-angle representation, with norms of $8.7 \mathrm{~mm}$ and 5.2 degrees.

While the prototypes here are meant to be representative of a solar array assembly mission, some limitations must be noted. An in-space assembly experiment will likely use Electron-beam welding, which requires a vacuum; hot-melt adhesive (HMA) is used in these trials as a welding analogue. Gravity affects the ground demonstrations and induces strains on the joints that will not be experienced in orbit. The LSMS is mounted to the ground, but will either be a mobile manipulator or attached to the spacecraft. All manipulators in a space mission will have onboard sensors, including computer vision and Light Detection and Ranging (LIDAR), permitting measurement of the structure by all robots, instead of relying on a single global metrology system. Onboard sensors must also handle a stark distinction between sunlit

\footnotetext{
${ }^{3} N$ is distinct from the part count due to intermediate steps.

${ }^{4}$ To distinguish the experimental LRM and DM from the concepts presented earlier, they will be referred to as the LSMS and IPJR.
}

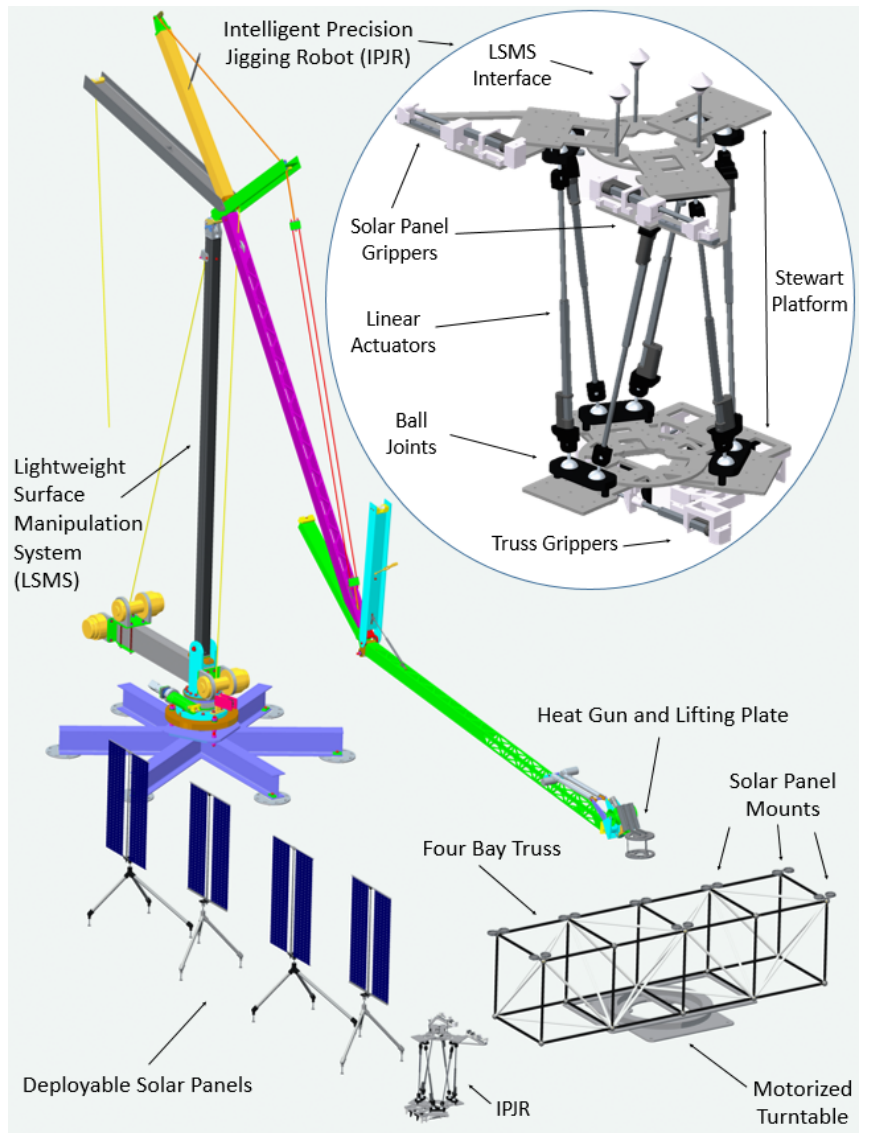

Fig. 4. Diagram showing workspace with the LSMS, IPJR, turntable, truss, and panels shown to relative size. Inset: IPJR detail.

and shaded components, which was not simulated in the laboratory. In-space assembly will employ several joining methods besides welding, including docking with interfaces allowing power, data, and fluid flow between components. In these trials, all robots are tethered to power supplies, but inspace robots may have their own power sources and charging stations.

\section{A. Solar Array Mockups}

The backbone truss was a 4-bay square truss with $1 \mathrm{~m}$ diagonals. Two of the ten top suface nodes were the mounting locations for the IPJR and were otherwise featureless. The eight other nodes featured a pair of $60 \mathrm{~mm}$ diameter flat plates, which are generalized assembly interfaces for the solar panels.

The grasping location of the solar panels for the IPJR was located at the top of the tripod base. Each solar panel leg of the tripod consisted of a threaded rod embedded in an HMA matrix to be melted during the welding analogue step. Each solar panel consisted of two rolled-up Mylar sheets attached by bars to a telescoping rod. The panel could be deployed by the LSMS hooking to the bar attached to the top of the telescoping rod, and pulling upward.

To simulate a six degree of freedom joint, the panel legs were not allowed to come into direct contact with the truss, and were instead nominally placed $10 \mathrm{~mm}$ above the surface 


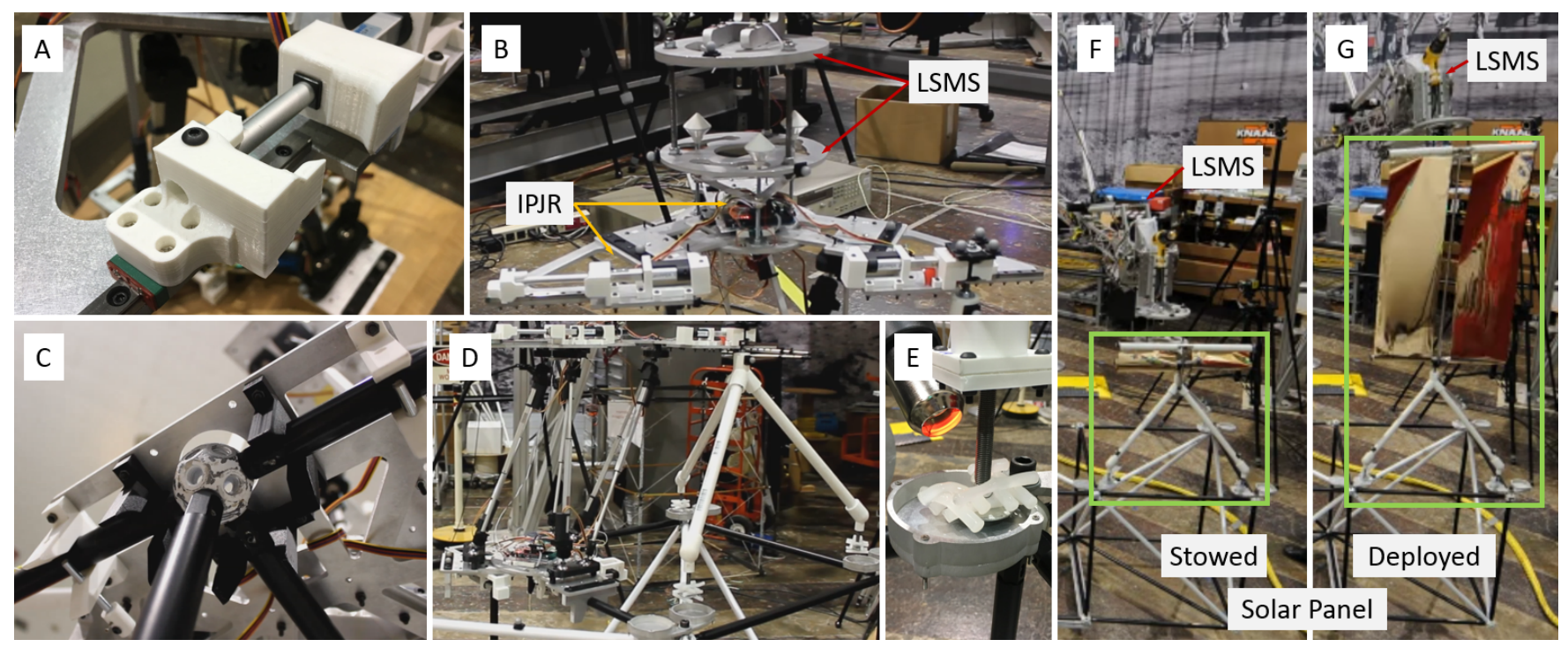

Fig. 5. A) Linear-actuated solar panel gripper on the IPJR; B) The IPJR locks onto the LSMS; C) The IPJR grasps the truss by locking three pins around three struts; D) the Stewart platform on the IPJR positions the solar panel legs over the truss aluminum plates; E) the LSMS joins a leg to the truss by melting HMA, forming a connection over the gap; F) the LSMS prepares to deploy the panel; and G) the LSMS finishes deploying the panel.

so that the welding analogue could fill the gap. The joints had to withstand both the weight of the panels and the deployment forces imparted by the LSMS.

\section{B. Assembly Robots}

Each assembly robot was an independent robot capable of receiving commands and returning feedback over radio to a central computer running the assembly algorithm.

The IPJR was designed to place panels over two bays while attached to a truss node located between the bays. This required the top plate to move up to $200 \mathrm{~mm}$ over each bay, and $100 \mathrm{~mm}$ vertically. A Stewart platform was designed to meet these goals, and could rotate more than 45 degrees on each axis. The IPJR employed two linear actuator grippers on its top plate to hold a panel over each bay, each with features to correct alignment as the gripper closed on the panel. The IPJR grasped the truss using brackets to guide the struts into the capture envelope, then locked onto the struts with extended pins. The IPJR achieved mobility with assistance by the LSMS. For this experiment, the IPJR to LSMS interface used gravity to capture conical IPJR posts into grooves on the end-effector.

The LSMS is a tendon-actuated arm with 4 links totaling 12.75 meters in length, and has a $500 \mathrm{~kg}$ lifting capacity. Its base joint rotates in the vertical axis; the remainder of the joints are parallel in the vertical plane, limiting the range of motion. While the LSMS can be mounted on a mobile base and is capable of hand-over-hand motion, in the laboratory is it fixed to the floor. A custom end-effector lifts the IPJR and performs the welding analogue by melting the HMA with hot air. To lift the IPJR, the LSMS grossly positions itself over the IPJR which then uses the dexterity of the Stewart platform to connect to the end-effector.

The turntable was a single degree-of-freedom robot that could rotate clockwise or counterclockwise at a given ve- locity. The turntable robot gave the assembly system the extra degree of freedom necessary to properly orient the truss during assembly procedures.

\section{Metrology}

Eight Vicon Bonita cameras were stationed around the truss and the panel storage location. Due to its size, only the LSMS end effector could fit within the workspace. Each tracked object had at least five $14 \mathrm{~mm}$ retroreflective markers spread over its visible surface. The bottom plate of the IPJR had eight markers due to it being partially obscured by the top plate. The standard deviations of the measurement noise of a still object were estimated to be $0.5 \mathrm{~mm}$ and 0.5 degrees in the translation and rotation axes.

\section{Assembly Sequence}

To join each panel to the truss, the following sequence is performed $^{5}$ :

1) IPJR begins at storage location.

2) IPJR grasps a panel (Figure 5A).

3) IPJR grasps LSMS and moves to truss (Figure 5B).

4) IPJR grasps truss and releases LSMS (Figure 5C).

5) IPJR positions the panel (Figure 5D).

6) LSMS joins legs to truss plates with turntable assistance (Figure 5E).

7) IPJR releases panel.

8) IPJR grasps LSMS and releases truss.

9) IPJR moves to storage location and releases LSMS.

After the last panel is fixed to the truss:

1) LSMS hooks a panel (Figure 5F).

2) LSMS deploys panel (Figure 5G).

${ }^{5}$ IPJR mobility is performed by the LSMS. 


\section{E. Algorithm Implementation}

A central computer operated the assembly algorithm in GNU Octave [25] at $5 \mathrm{~Hz}$. The slow pace of the assembly sequence did not require a higher frequency.

Goal has two different implementations. For gripper operation and joining, the actions were performed for a set amount of time before returning True. Gripper failures were not observed. For all other motions, Goal compared relative positions between objects; if the relative position and orientation is within the permissible error boundaries on all six axes, a 5 second timer begins to prevent false positives through jitter. If error remains bounded after 5 seconds, Goal returns True. Teleoperation was necessary to train the relative positions and orientations for objects when in their goal poses. Each step had an acceptable per-axis translation error ranging from 3-5 mm and 3-10 degrees. The panel-totruss assembly step had an acceptable error of $5 \mathrm{~mm}$ and 3 degrees on each axis, the norms of which were $8.7 \mathrm{~mm}$ and 5.2 degrees. All robots were capable of sub-millimeter precision at a significant time penalty.

The Measure function recorded Vicon position and rotation data for each object in the scene: each plate of the IPJR, each panel, the truss, and the end effector of the LSMS. The Vicon tracker lacks an onboard state transition function, meaning that an additional filter making use of a transition function could improve the estimate. Filter implements the extended Kalman filter to improve the estimates of the positions and rotations of each object given its current estimate, covariance, and controls. The control vector is used to predict where the objects would have moved, including parts affixed to moving robots. Still objects are assumed to stay still, and are given very small standard deviations: $0.1 \mathrm{~mm}$ and 0.1 degrees, small enough to prevent measurement jitter from steering the estimate too much, while large enough to prevent singularities in the filter calculation. The filter favors measurements for moving objects to account for variations in motor output, assigning transition standard deviations of 4 $\mathrm{mm}$ and 4 degrees. The Vicon measured complete states for all parts and most robots. However, the Vicon could measure only the end-effector on the LSMS; the remainder of the links were estimated from a state model.

The Command function has distinct implementations for each of the three robots in the workspace. The turntable used saturated proportional control to rotate, slowing proportionally within 5 degrees of the goal. The LSMS implementation used saturated proportional control to drive each individual motor, also slowing proportionally within 5 degrees of the goal. The IPJR implementation used the inverse Jacobian of plate motion to plan a linear path between the current state and the desired state, and required one of the two plates to be fixed and the other to be free, so that the system was not underactuated. Saturated proportional control started slowing the IPJR within $20 \mathrm{~mm}$ of its goal.

\section{Assembly Trial Results}

The assembly sequence for an individual panel was performed nine times, consisting of two complete solar array
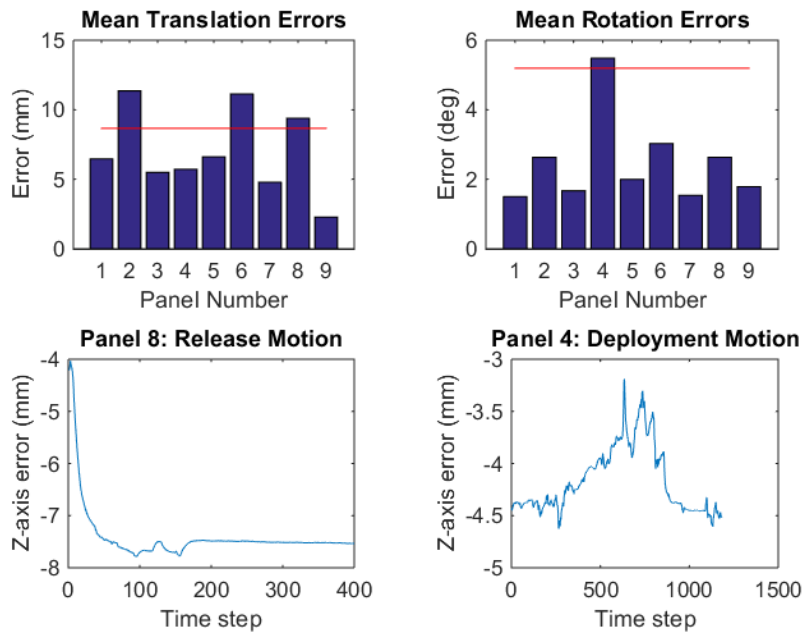

Fig. 6. Top: total translation and rotation errors for the nine test panels, when measured after the IPJR releases the newly-fixed panels, shown with the total acceptable errors of $8.7 \mathrm{~mm}$ and 5.2 degrees. Bottom: two cases demonstrating (left) the vertical sagging of panel 8 under its own weight after being released, adding to the total error, and (right) the vertical strain placed on the joints of panel 4 by the LSMS deploying the panel.

assembly trials and a single panel assembly trial.

The primary goal of joining all panels to the truss and deploying all panels was successful for all nine panels. The secondary goal was to ensure each solar panel was positioned within $8.7 \mathrm{~mm}$ and 5.2 degrees. These errors were measured after the IPJR released the panels to allow the panels to settle. The results are shown in the top row of Figure 6. Six of the nine panels remained within the total acceptable bounds. Panels 2, 6, and 8 exceeded the allowable translation error, and panel 4 exceeded the allowable rotation error. Three major contributing factors were identified:

- The quality of each joint was sensitive to errors in the heat gun positioning, resulting in joints with variable stiffness as the HMA often flowed too much or too little. Similar errors could occur with in-space welding.

- Command stopped moving the panels as soon as it reached the error bounds, placing it near the acceptable limits. This behavior can be corrected.

- The weight of the panels compressed the joints after the IPJR released the panels. This is an artifact of the ground-based assembly process.

The latter two of these factors can be seen in the lower left plot in Figure 6 for panel 8; prior to release at time step 0 , the Z-axis error was within the $5 \mathrm{~mm}$ limit, but $4 \mathrm{~mm}$ below the goal. It then dropped to over $7 \mathrm{~mm}$ below the goal after the IPJR removed its support.

The flexibility of the joints was observed during the deployment phase. The friction in each panel's telescoping rod contributed most of the resistance, combined with lateral forces since the LSMS did not perfectly follow a vertical path. The panels shifted upward between 0.5 and $1 \mathrm{~mm}$ typically, then rebounded to the original position. The vertical motion of panel 4 is shown in Figure 6. 


\section{DiscuSSION AND CONCLUSION}

To be practical, autonomous robotic assembly will require many large leaps, including support for numerous joining methods, the means to accurately estimate the state of the structure including any errors that may be present, the use of several different kinds of assembly robots cooperating to assemble parts, and the ability for the agents to adapt to unforeseen circumstances. Many of these methods correspond to ongoing challenges in mobile robotics, namely in the fields of estimation, event and error detection, sequence planning and replanning, and robust manipulation.

The overall goal of this research is to make autonomous assembly more robust using representative parts and joining techniques that are more likely to be used in practice. Event detection, error correction, and sequence replanning are key to its success. This paper presented the early results of this research, showing that, with an assembly algorithm using state estimation:

- Mobile robots can join parts to each other to a high degree of accuracy relying on state estimation instead of precise mechanical interfaces.

- A Stewart platform can serve as a mobile manipulator with high precision and stability.

- A team consisting of the Stewart platform and a tendonactuated, long reach manipulator can collaborate to assemble a structure and manipulate it afterward.

These trials revealed shortcomings that will be addressed in future work. Onboard sensing will better inform state estimation and identify assembly mistakes, replacing the Vicon system. Off-the-shelf Stewart platforms capable of sub-micron precision can improve assembly accuracy by a factor of 1000. Welding will replace HMA. Machine learning techniques will be trained by simulations and physical trials to recognize errors and to plan repair sequences. Progress in all these areas will make autonomous assembly practical, with direct application to space structures such as solar arrays and space telescopes, and extending to other structures.

\section{ACKNOWLEDGMENT}

This research was funded by the NASA Langley Research Center Innovation Fund.

\section{REFERENCES}

[1] W. K. Belvin, W. R. Doggett, J. J. Watson, J. T. Dorsey, J. Warren, T. C. Jones, E. E. Komendera, T. Mann, and L. Bowman, "In-Space Structural Assembly: Applications and Technology," in 3rd AIAA Spacecraft Structures Conference, 2016, p. 2163.

[2] J. Dorsey and J. Watson, "Space Assembly of Large Structural System Architectures (SALSSA)," in AIAA SPACE 2016, 2016, p. 5481.

[3] H. Ardiny, S. Witwicki, and F. Mondada, "Construction Automation with Autonomous Mobile Robots: A Review," in 3rd RSI International Conference on Robotics and Mechatronics (ICROM). IEEE, 2015, pp. 418-424.

[4] J. T. Dorsey, W. Doggett, E. Komendera, N. Correll, R. Hafley, and B. King, "An Efficient and Versatile Means for Assembling and Manufacturing Systems in Space," in Proceedings of the AIAA SPACE Conference, 2012, pp. 1-19.

[5] E. Komendera, J. T. Dorsey, W. R. Doggett, and N. Correll, "Truss Assembly and Welding by Intelligent Precision Jigging Robots," in 2014 IEEE International Conference on Technologies for Practical Robot Applications (TePRA). IEEE, 2014.
[6] E. Komendera and N. Correll, "Precise Assembly of 3D Truss Structures using MLE-Based Error Prediction and Correction," The International Journal of Robotics Research, 2015.

[7] Q. Lindsey and V. Kumar, "Distributed Construction of Truss Structures," in Algorithmic Foundations of Robotics X. Springer, 2013, pp. 209-225.

[8] S. R. Barros dos Santos, S. N. Givigi, and C. L. Nascimento, "Autonomous Construction of Structures in a Dynamic Environment Using Reinforcement Learning," in Systems Conference (SysCon), 2013 IEEE International. IEEE, 2013, pp. 452-459.

[9] M. Vona, C. Detweiler, and D. Rus, "Shady: Robust Truss Climbing with Mechanical Compliances," in Experimental Robotics. Springer, 2008, pp. 431-440.

[10] F. Nigl, S. Li, J. E. Blum, and H. Lipson, "Structure-Reconfiguring Robots: Autonomous Truss Reconfiguration and Manipulation," IEEE Robotics \& Automation Magazine, vol. 20, no. 3, pp. 60-71, 2013.

[11] J. Worcester, R. Lakaemper, and M. A. Hsieh, "3-Dimensional Tiling for Distributed Assembly by Robot Teams," in Proceedings of the 13th International Symposium on Experimental Robotics (ISER2012). Springer, 2012, pp. 143-154.

[12] N. Napp and R. Nagpal, "Distributed Amorphous Ramp Construction in Unstructured Environments," Robotica, vol. 32, no. 02, pp. 279290, 2014.

[13] V. Helm, S. Ercan, F. Gramazio, and M. Kohler, "Mobile Robotic Fabrication on Construction Sites: DimRob," in 2012 IEEE/RSJ International Conference on Intelligent Robots and Systems (IROS). IEEE, 2012, pp. 4335-4341.

[14] F. Augugliaro, A. Mirjan, F. Gramazio, M. Kohler, and R. D’Andrea, "Building Tensile Structures with Flying Machines," in 2013 IEEE/RSJ International Conference on Intelligent Robots and Systems (IROS). IEEE, 2013, pp. 3487-3492.

[15] R. Madhavan, "The 2016 Airbus Shopfloor Challenge [Competitions]," IEEE Robotics Automation Magazine, vol. 23, no. 3, pp. 21-22, Sept 2016.

[16] B. Hamner, S. Koterba, J. Shi, R. Simmons, and S. Singh, "An Autonomous Mobile Manipulator for Assembly Tasks," Autonomous Robots, vol. 28, no. 1, pp. 131-149, 2010. [Online]. Available: http://dx.doi.org/10.1007/s10514-009-9142-y

[17] M. Dogar, R. A. Knepper, A. Spielberg, C. Choi, H. I. Christensen, and D. Rus, "Towards Coordinated Precision Assembly With Robot Teams," in Experimental Robotics. Springer, 2016, pp. 655-669.

[18] R. Simmons, S. Singh, D. Hershberger, J. Ramos, and T. Smith, "First Results in the Coordination of Heterogeneous Robots for Large-Scale Assembly," in Experimental Robotics VII. Springer, 2001, pp. 323332.

[19] A. Stroupe, T. Huntsberger, A. Okon, and H. Aghazarian, "Precision Manipulation with Cooperative Robots." Springer, 2005, pp. 235248.

[20] W. Doggett, "Robotic Assembly of Truss Structures for Space Systems and Future Research Plans," in Aerospace Conference Proceedings, 2002. IEEE, vol. 7. IEEE, 2002, pp. 3587-3598.

[21] I. Bonev, "Delta Parallel Robot: The Story of Success," Newsletter, available at http://www.parallelmic.org, 2001.

[22] W. Doggett, J. Dorsey, T. Collins, B. King, and M. Mikulas, "A Versatile Lifting Device for Lunar Surface Payload Handling, Inspection \& Regolith Transport Operations," in Space Technology and Applications International Forum-STAIF 2008, vol. 969, 2008, pp. 792-808.

[23] National Institute of Aerospace, "BIG Idea Challenge: 2017 Competition," http://bigidea.nianet.org/past-competition-themes/2017competition/, Accessed: 2017-02-17.

[24] Vicon, "Motion Capture Systems - Vicon," http://www.vicon.com, accessed: 2016-07-20.

[25] J. W. Eaton, D. Bateman, S. Hauberg, and R. Wehbring, GNU Octave Version 4.0.0 Manual: A High-Level Interactive Language for Numerical Computations, 2015. [Online]. Available: http://www.gnu.org/software/octave/doc/interpreter 